\title{
Une poétique du désenchantement
}

La rose et le crapaud, signes panériens d'une poésie perdue

\section{Lina Iglesias}

\section{(2) OpenEdition}

1 Journals

Édition électronique

URL : http://journals.openedition.org/agedor/687

DOI : 10.4000/agedor.687

ISSN : 2104-3353

Éditeur

Laboratoire LISAA

Référence électronique

Lina Iglesias, "Une poétique du désenchantement », L'Âge d'or [En ligne], 7 | 2014, mis en ligne le 01

mars 2014, consulté le 01 mai 2019. URL : http://journals.openedition.org/agedor/687 ; DOI :

10.4000/agedor.687

L'Âge d'or. Images dans le monde ibérique et ibéricoaméricain 
Lina IGLESLAS

Université Paris Ouest Nanterre La Défense, EA 369 Études Romanes

\title{
Une poétique du désenchantement : la rose et le crapaud, signes panériens d'une poésie perdue
}

\begin{abstract}
Résumé : Le deuxième volume des œuvres poétiques de L. M. Panero, édité par Túa Blesa, coïncide avec un changement géographique : quittant la péninsule, le poète trouve dans les îles Canaries un espace autre pour vivre, bien que toujours interné, et écrire comme en témoigne l'intense production de ces dernières années. Néanmoins, ces poèmes mettent en évidence une inflexion dans l'écriture panérienne, marquée à présent par un ressassement qui confine le poète dans un espace poétique mortifère. La rose, et le crapaud, signes contraires mais complémentaires sont le signe de ce désenchantement poétique.
\end{abstract}

Mots-clés : Leopoldo María Panero - œuvre 2000-2010 - rose - crapaud - poétique désenchantement - mort

Resumen: El segundo volumen de las obras poéticas de L. M. Panero, editado por Túa Blesa, corresponde con un cambio geográfico: al dejar la península, el poeta halla en las islas Canarias un espacio diferente para vivir, aunque en un manicomio, y escribir como lo recalca su intensa producción poética de esos últimos años. Sin embargo, estos poemas revelan una inflexión en la escritura paneresca, marcada por un proceso de repetición obsesivo que encierra al poeta en un espacio poético mortífero. Tanto la rosa como el sapo, signos contrarios pero complementarios son el signo de este desencanto poético.

Palabras clave: Leopoldo María Panero - obra 2000-2010 - rosa - sapo - poética - desencanto muerte

$\mathrm{E}$ n 2012, Túa Blesa, l'un des premiers critiques espagnols à avoir écrit sur l'œuvre poétique de Leopoldo María Panero, réunit à nouveau les poèmes du poète dans un deuxième volume intitulé Poesía completa (2000-2010), aux éditions Visor de Poesía, volume qui fait suite à celui qui rassemblait les recueils écrits entre 1970 et 2000. Ce deuxième tome de presque six cents pages témoigne non seulement d'un singulier dynamisme créateur et d'une puissante nécessité d'écrire de la part du poète mais nous permet surtout de prendre conscience de la constance de cette écriture, de sa régularité puisqu'elle s'étend sur plus de quarante années. Au-delà des coïncidences dues aux hasards des publications, ces deux volumes semblent mettre en lumière non pas une véritable rupture entre deux temps de la production panérienne, mais une sorte de tournant, ou plutôt d'inflexion de cette poésie. En outre, ce deuxième tome correspond aux poèmes composés, à quelques exceptions près, dans l'unité psychiatrique de l'Hôpital Juan Carlos I de Las Palmas de Gran Canaria, où Leopoldo María Panero a passé ses dernières années, après avoir été interné à sa demande, en 1997 à l'Hôpital Psychiatrique insulaire de Las Palmas pour ne plus rester dans l'Hôpital psychiatrique San Juan de Dios, plus connu 
sous le nom de «manicomio de Mondragón », qui renvoie au recueil publié en $1987^{1}$ : ce changement géographique, mais pas vraiment spatial dans la mesure où il s'agit toujours d'un «asile », d'un lieu psychiatrique, s'accompagne ainsi d'une volonté personnelle de se déplacer, dont il faudrait pouvoir néanmoins connaittre les tenants et les aboutissants, mais cela dépasse le cadre de cette intervention. L'on ne peut s'empêcher aussi de voir une autre coïncidence, cette fois géographique ; bien que formant partie du territoire espagnol, les Canaries n'en sont pas moins des îles, et éloignées du continent. Changer d'hôpital implique aussi sortir d'un territoire marqué par une identité nationale et une histoire familiale : une distance physique et concrète s'avère nécessaire, comme si le poète cherchait ailleurs un lieu à habiter. De par sa configuration, l'île suppose à son tour un rapport au monde différent, un lien interrompu avec les autres qui appartiennent au passé : poète dit maudit, banni de la société, Leopoldo María Panero choisit cette fois d'exclure d'une certaine façon la société, de la marginaliser en s'en séparant, en s'en détachant comme l'on coupe le lien ombilical qui étrangle. Mais de par sa topographie, l'île se présente aussi comme le lieu des contradictions : si elle ouvre sur un horizon infini, elle délimite en même temps un espace circonscrit qui renforce l'impression d'isolement.

À partir de ce constat biographique, il apparait que les recueils écrits aux Canaries s'inscrivent dans un même mouvement, certes légèrement amorcé auparavant, mais qui ne cesse de s'accentuer : confronté à un enfermement - peut-être relatif dans la mesure où il peut sortir, rencontrer des gens, participer, en étant accompagné, à des colloques...-, le poète entretient avec la poésie et l'acte d'écrire une relation forte, exacerbée qui lui permet de contrer le suicide : "la escritura me permite no suicidarme »" Si l'écriture comble le temps et le vide existentiel, elle le renvoie néanmoins à sa condition, l'enfermant finalement dans un espace, celui de la poésie, matérialisé par l'espace blanc de la page, dont il ne parvient plus à sortir.

L'isolement vécu comme un esseulement qui se charge d'un poids existentiel génère une écriture qui se replie sur elle-même, suivant des circonvolutions métaphoriques dont témoigne la prégnance d'images, mots, motifs ou paroles dans l'œuvre panérienne. Une telle persistance, qui pourrait s'analyser à partir des procédés de répétition, variation, déclinaison renforce l'idée que l'écriture est devenue le seul mode d'exister, de continuer d'être en dépit de tout, mais en même temps, le moi, si tant est que l'on puisse encore se prévaloir de cette notion pour identifier celui qui est à l'œuvre dans les poèmes panériens, se morfond dans un espace de réclusion délimité par le propre espace poétique. C'est à travers l'analyse de deux motifs, la rose et le crapaud, que nous allons tenter de comprendre comment opère, dans l'écriture, la tension entre vivre et écrire.

Parmi les motifs et images omniprésents dans l'œuvre de Leopoldo María Panero s'imposent depuis les premiers recueils la rose et le crapaud : éléments antithétiques par excellence, qui s'excluent et se rejettent tout en appartenant au même territoire imaginaire, la rose et le crapaud ponctuent à présent les poèmes panériens comme signes d'une poétique perdue et désenchantée. Ils envahissent l'espace poétique pour dire ce qui n'a pas eu lieu, notifiant ainsi un creux. Dans le corpus que nous avons délimité - Poesía completa (2000-2010) $)^{3}$, et au fil des poèmes, plutôt courts mais qui se font écho, un singulier paysage de ruines poétiques semble s'esquisser, habité par quelques motifs rappelant à leur manière, les vanités.

\footnotetext{
1 PANERO, Leopoldo María, Poemas del manicomio de Mondragón, Madrid, poesía Hiperión, 1987.

2 http:/ / elultimodiadesocrates.wordpress.com/2013/01/02/leopoldo-maria-panero-poesia-contra-locura-2/

${ }^{3}$ PANERO, Leopoldo María, Poesía completa (2000-2010), ed. de Túa Blesa, Madrid, Visor, 2012.
} 


\section{De la Rose à la rose}

Topique des topiques, la rose jaillit d'un lointain passé poétique drainant dans l'espace du poème de multiples résonances; mot chargé de voix autres, de vers célèbres tapis dans la mémoire de chacun, la rose porte en elle un potentiel poétique qu'un emploi abondant en littérature et en poésie a certes un peu fané, mais qui demeure dynamique. De par justement son ancrage dans la mémoire poétique, elle ne cesse d'avoir un fort pouvoir évocateur. Mais la rose panérienne échappe à ses connotations traditionnelles: loin du Carpe Diem, loin de l'absente du bouquet mallarméen ou de la rose métapoétique de Gertrude Stein - « Rose is a rose is a rose is a rose »-, la rose panérienne apparait pour dire ce qui ne peut plus advenir. Dans un article consacré aux fleurs et au don qu'elles supposent comme offrande et geste lyrique, Dominique Rabaté définit, à partir d'une lecture des poèmes de Philippe Jaccottet et Francis Ponge, la portée des fleurs dans la poésie contemporaine: "le mimosa ou la pivoine emblématisent, dans leur singularité, le mystère même de la présence au monde et le poème devient méditation sur les moyens que l'écrivain peut susciter, qu'il doit utiliser pour accueillir pleinement ce que lui donnent les fleurs » ${ }^{4}$. Et il conclut ainsi sur « le mystère d'une apparition [...], ce que nous offre alors la poésie, c'est une épiphanie profane du monde $»^{5}$, épiphanie que font éclore les pivoines dans le poème de Philippe Jaccottet. Néanmoins cette dimension heureuse de la fleur qui permet d'entrouvrir un passage vers un autre monde, aussi éphémère soit-il, ne correspond pas au processus créateur panérien. Lorsque parait la rose dans le poème, elle condense en elle des significations oubliées qui ne peuvent plus affleurer dans l'espace poétique; le mot demeure tourné non plus vers une possible rencontre mais vers lui-même, se repliant sur une absence. Lors de sa manifestation dans le vers, la rose apparait seule, isolée, comme dépossédée de son passé ; à la lecture, l'on est saisi par la sécheresse ou l'aridité qui entoure le mot, dépouillant ainsi la rose de tout écho. Aucun déploiement, aucun épanouissement ne lui semble permis : plus qu'une image, c'est d'un simple mot dont il s'agit, livré dans un dénuement extrême. En effet, la rose ne donne pas lieu à une expansion poétique : guère d'adjectifs, pas de ramification syntaxique ou métaphorique qui viendraient redonner à la rose une autre dynamique, un autre souffle poétique. Lorsqu'un adjectif vient qualifier la fleur, ce n'est que pour mieux affirmer son impuissance ou son pouvoir mortifère ${ }^{6}$ : rosa cruel de la creación ${ }^{7}$ rosa cadavérica, rosa pálida. Le poème s'ouvre comme un espace non pas d'accomplissement ou de réalisation poétique mais au contraire d'anéantissement.

Néanmoins, dans la condensation et la fulgurance du poème, la rose ouvre un léger interstice; alors que la gamme chromatique se voit réduite à deux ou trois couleurs récurrentes - le bleu, le jaune mais très ponctuellement, et par connotation le blanc que renvoie le mot «página »-, la rose quant à elle suggère un écho chromatique qui vient en contrepoint du noir imposé par l'encre qui porte les vers. Traditionnellement, cette couleur peut prendre le sens d'une possible régénération, mais dans l'espace poétique panérien, elle reste une touche qui s'efface aussitôt apparue. Si, dans le sillage de Dominique Rabaté et d'autres critiques, l'on considère que la parole poétique dépend de sa valeur performative?,

\footnotetext{
${ }^{4}$ RABATÉ, Dominique, Gestes lyriques, Paris, Éditions Corti, Les Essais, 2013, p. 124.

${ }^{5}$ Idem, p. 137.

${ }^{6}$ Un recueil posthume de poèmes inédits vient d'être publié sous le titre significatif de Rosa enferma, Madrid, Huerga \ Fierro, 2014.

7 PANERO, Leopoldo María, Danza de la Muerte (2004), in Poesía completa (2000-2010), op. cit., p. 232

8 PANERO, Leopoldo María, Buena Nueva del desastre (2002), ibid, p. 128.

${ }^{9}$ " le langage accomplisse réellement quelque chose » RABATÉ, Dominique, op. cit., p. 21.
} 
on en vient donc à s'interroger sur le sens de cette écriture qui semble vouée au néant. La rose apparaît ainsi comme le signe d'une poétique perdue, oubliée car celle-ci n'a plus de sens ni de place aujourd'hui; déjà dans les recueils précédents, la rose symbolisait l'impossible relation entre les hommes et la création, entre la cité et le poète : El destino de los hombres es arrojar piedras a la rosa ${ }^{10}$. À présent, au-delà de la disparition de la parole poétique, la rose perdure comme vestige :
Nunca supe lo que el nardo era
nunca supe qué fue la rosa
quizás la flor, quizás la tarde
que en la tarde se deshoja
buscando aún el laurel invertido
la rosa que en la rosa se deshoja. ${ }^{11}$

Dans ces vers où le moi se remet en question en tant que poète, car il ignore le sens à jamais perdu des fleurs qui appartiennent, entre autres, au registre poétique, l'interrogation se centre sur la rose, objet du poème, qui finit par s'étioler, se faner dans le dernier vers délimitant ainsi symboliquement l'espace poématique. Face à la certitude du moi quant à son statut de poète qu'il nie de façon catégorique par l'anaphore de - nunca supe -, domine le doute sur l'objet poétique que représente la rose, objet inaccessible mais a-t-il vraiment un jour existé. Dans le dernier vers, le moi n'est plus que locuteur pour dire la décomposition de la rose: l'assonance en $[\mathrm{o} / \mathrm{a}]$ qui parcourt ce vers, accentuée par l'allitération en $[\mathrm{s}]$ fait de la rose un son, un «bruissement de la langue » pour reprendre le titre de Roland Barthes ${ }^{12}$, lointain écho de ce qu'elle fut dans sa plénitude poétique. Mais ce temps suppose de remonter à la poésie des troubadours, comme le suggère l'épigraphe de ce poème : "Er resplan la flors enversa » Raimbaut d'Aurenga. La référence au plus ancien troubadour de Provence, représentant du «bel saber» au XII ${ }^{\mathrm{e}}$ siècle, pose d'emblée l'origine inimitable de la rose ; si le long poème de Raimbaut d'Orange creuse le mystère de la fleur " enversa ${ }^{13}$, les vers de L. M. Panero en marquent sa disparition. C'est pourquoi, après cet acmé de la rose lyrique, les poètes, les uns après les autres, se sont vus confrontés à l'impossible dire de la rose, comme en témoigne la présence récurrente dans l'œuvre de L. M. Panero des poètes qui ont cherché à découvrir le sens de cette fleur. Soit la rose « rilkiana » qui accompagne le poète vers la mort :

\author{
Ah la rosa del texto \\ la rosa hambrienta de tu sexo \\ húmedo de lágrimas y mojado \\ por el estiércol, por el estiércol \\ como una bendición \\ como tierra \\ para el sepulcro \\ ah la rosa infiel de la nada \\ la rosa
}

\footnotetext{
${ }^{10}$ PANERO, Leopoldo María, Poemas del manicomio de Mondragón, op. cit., p. 17.

${ }^{11}$ PANERO, Leopoldo María, Aguila contra el hombre, in Poesía completa (2000-2010), op. cit., p. 98.

12 BARTHES, Roland, Le bruissement de la langue. Essais critiques IV, Paris, Seuil, 1984.

${ }_{13}$ Voir au sujet de ce célèbre poème, l'article de STANESCO, Michel, « La fleur inverse et la « belle folie » de Raimbaut d'Orange », in Cabiers de civilisation médiévale, 1997, vol. 40, n 40-159, p. 233-252.
} 


\author{
mojada por las lágrimas \\ como el poeta aquel \\ al que mató una rosa ${ }^{14}$
}

Quelque temps avant de mourir en 1926, Rilke s'installe dans le Valais où il achève ses recueils les plus célèbres - Les Élégies de Duino et Les Sonnets à Orphée -, mais aussi Les Roses en français (paru en 1927) et demande d'inscrire comme épitaphe sur sa tombe: Rose, ô pure contradiction, désir / de n'être le sommeil de personne sous tant de / paupières.

C'est aussi, entre autres, la rose de Yeats : Ob labio oscuro de la rosa $[. .$.$] -ab Yeats lo dijo$ / enseñando una rosa secreta / a la manada ${ }^{15}$. Ou celle de Baudelaire : Rosa de las tinieblas y el grito / Flor del mal ${ }^{16}$. Mais pour L. M. Panero, la rose a définitivement perdu son enchantement: La rosa hoy se marchita / y cae sobre el papel / y una rosa murmura en mi oído : tú eres una rosa / tú eres menos que nada ${ }^{17}$. Le passage de l'article défini à l'article indéfini (la rosa / una rosa) témoigne du changement de statut du motif poétique et en s'effaçant, la rose entraine dans sa chute le moi poétique. D'où le glissement de la rose à la fleur, les fleurs : ayant perdu le sens originel de la rose, le poète a recours au terme générique. Ce mot vient en contrepoint de celui de la rose, et ponctue régulièrement les poèmes : Y un moribundo grita : ¿ ¿Qué es la rosa? I ¿Cuál es el secreto inmundo de la flor / Y de la vida herida? / De la ruina del papel, siempre en vano / Del jardín siempre en vano / De la flor / De la derrota eterna del poema / De la flor que cae vencida por la llama ${ }^{18}$.

Dans sa situation de dissonance par rapport au monde, le poète trouve dans la rose l'espace d'une contemplation mélancolique. Peut-être sera-t-on un peu surpris par cette lecture dans la mesure où dans la poésie de la première période, le ton contestataire et la portée souvent blasphématoire des poèmes définissent une poétique à la marge. Le sentiment de mélancolie témoigne de cette inflexion dans l'écriture panérienne :

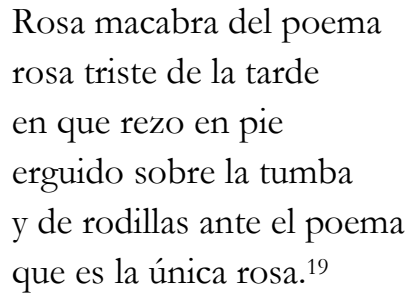

Dans ce court poème, la rose élégiaque se voit contaminée par un sentiment de mort qui est à l'œuvre dans l'écriture: l'emploi de l'antépiphore délimite un espace d'enfermement suscité par le ressassement du mot «rosa ». On notera que dans les deux premiers vers, l'adjectif postposé qui sert à caractériser la rose (macabra / triste) correspond à une tentative du locuteur de définir l'objet, de lui donner encore un sens alors que dans le derniers vers, l'antéposition de l'adjectif souligne son essence irréductible, accentuée par l'article défini : l'équivalence trouvée entre le poème et la rose permet de faire éclore le mot à la fin du poème, mais il est objet de recueillement. Retranché dans une

\footnotetext{
14 PANERO, Leopoldo María, Buena Nueva del desastre, in Poesía completa (2000-2010), op. cit., p. 130.

15 PANERO, Leopoldo María, Erección del labio sobre la página (2004), ibid, p. 200.

${ }^{16}$ Idem, p. 179.

17 PANERO, Leopoldo María, Poemas para un suicidamiento, ibid, p. 106.

18 PANERO, Leopoldo María, XXIV, Reflexión (2010), ibid, p. 560.

19 PANERO, Leopoldo María, Abismo (1999), in Poesía completa (2000-2010), op. cit., p. 45.
} 
solitude intérieure, le poète fait de la poésie et d'une littérature qu'il a fait sienne, un lieu à habiter; aussi sa voix se mêle-t-elle à d'autres voix poétiques, les vers des uns et des autres s'entrecroisant avec les siens. Si, auparavant les jeux d'intertextualité étaient plus marqués et renvoyaient à une pratique commune aux Novísimos, dorénavant le moi poétique convoque des vers ou des paroles comme commencement ou prolongement de sa propre parole, tissant ainsi une sorte de fil invisible entre des poétiques différentes; parfois fondus dans le corps même du texte panérien, parfois mis à distance au moyen de l'expression « lo dijo » ou «como lo dijo », insistant ainsi sur l'autorité poétique dont il se revendique pour mieux la remettre en question et la désacraliser. Mais allant jusqu'à se citer : "Panero lo dijo », ou en se désignant comme poète mort dont la parole peut être citée : "Panero lo dijo antes de morir $»^{20}$. Tout a été dit, tout a déjà été écrit et à présent le poète, en rappelant les vers des autres poètes de façon précise ou incertaine, signifie la vanité d'une nouvelle parole. De même, par leur présence abondante, les poètes qu'il ne cesse de nommer en les égrenant au fil des poèmes, dans une sorte de litanie, - Mallarmé, Yeats, Goethe, Gamoneda, ...-, deviennent les interlocuteurs du seul dialogue possible pour lui : devenu difficile et fragmentaire à l'extérieur, le rapport au réel ne passe que par la médiation de ses semblables dans l'espace du poème qui l'enferme davantage.

Cette solitude que les autres voix poétiques tentent de combler, renforce le sentiment de mélancolie qui caractérise le moi panérien dans les recueils retenus, et dont la rose est la manifestation. Dès le premier recueil, Así se fundó Carnaby street, étaient visibles les signes de ce deuil impossible qui perdure, mais ils renvoyaient au temps de l'enfance qui, fût-elle cruelle et dévastatrice, fait défaut. Au-delà des jeux ou pratiques poétiques propres aux Novísimos $^{21}$, les références à l'enfance dans ce recueil matriciel qui ouvre une longue trajectoire poétique, constituent un territoire poétique fondateur, placé sous le signe d'une blessure originelle qui persiste jusque dans les derniers recueils.

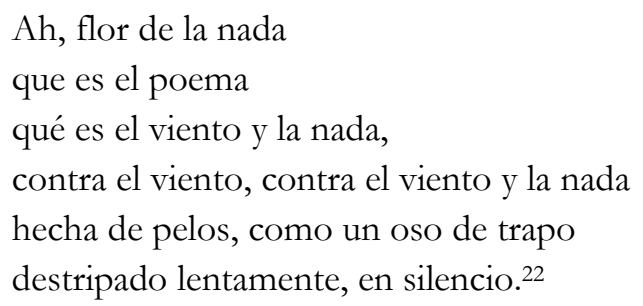

Dans un face-à-face avec les mots qui signifient le néant, le moi panérien semble aux prises avec un désespoir qui donne lieu au ressassement; dans le tourbillon créé par les jeux de répétition, la fleur se rapproche de l'ours en peluche, signe dérisoire et usé de l'enfance lointaine. De façon ponctuelle, l'image de l'enfant surgit encore dans certains poèmes, mais cette fois en creux, à peine visible, figure spectrale d'un moi égaré :
Ah tiniebla del ser, flor de la nada
flor que habita las tinieblas
silueta azul de la nada
flor contra el espanto

\footnotetext{
${ }^{20}$ PANERO, Leopoldo María, Reflexión (2010), ibid, p. 558.

21 ZIMMERMANN, Marie-Claire, «Les jeux de la pratique et de la théorie dans la poésie actuelle espagnole (1965-1981), in Les langues néo-latines, 1983, n²45, pp. 5-26.

22 PANERO, Leopoldo María, «Infancia », Erección del labio sobre la página (2004), in Poesía completa (2000-2010), op. cit., p. 193.
} 


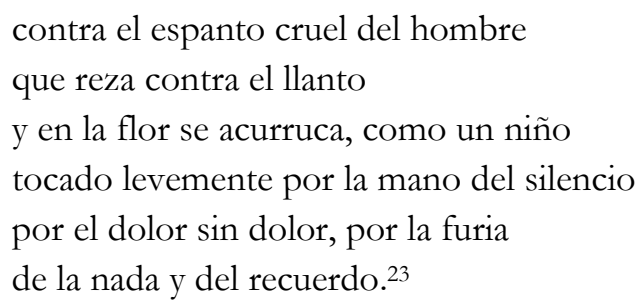

Dans ces vers, les figures de l'homme et de l'enfant surgissent presque en même temps, elles s'appellent mutuellement mais dans un mouvement de régression l'enfant occupe le deuxième temps du poème : ces vers ferment le poème dans une sorte d'apodose qui notifie un repliement du moi poétique. Du souvenir on ne saura rien, car il est vide, et le silence a pris la place d'un dieu créateur. La perte liée à l'enfance qui marquait initialement le processus créateur se prolonge jusque dans les œuvres récentes, mais opérant un glissement vers la perte poétique : aussi retrouve-t-on encore disséminés au fil des recueils les personnages de Pulgarcito ${ }^{24}$, Garbancito, et autres, mais ils émergent du lointain imaginaire enfantin pour désigner l'impuissance :

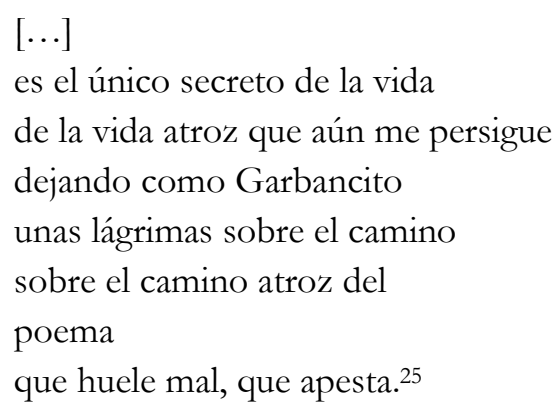

Ici, les petits cailloux sont devenus des larmes qui traduisent la douleur, larmes que les premiers vers ont convoquées à travers la figure du poète Mallarmé : Mallarmé, Ma larme I mi lágrima que no es nada. Le glissement sémantique entre le nom et le substantif permet au poète de s'approprier un écho - «mi »-, mais il reflète le passage du poétique au néant, à la stérilité. Si « Ma larme » pouvait encore avoir, écrite dans une autre langue - le français avec une typographie différente et une majuscule, une noble résonance poétique, digne des Romantiques, le simple mot « lágrima », issu d'un deuxième glissement, de langue cette fois, perd tout statut poétique et renvoie à la peine infime et vaine de l'être. De fait, les cailloux ne ramènent plus à la maison mais au poème, lieu de mort : y el poema / sea un blanco sudario I sea blanco como la nada.

En lisant les poèmes, on a le sentiment qu'il est difficile d'écrire car la parole poétique se dérobe, d'où la répétition, l'obsession, le recommencement à l'identique... tourner autour du même mot, ou de la même image et s'y laisser enfermer. L'image omniprésente de la chute témoigne de l'échec de l'acte créateur : tout tombe, chute, comme soumis à une loi interne au poème qui empêche les mots de rester en suspens, ou de s'épanouir. Comme l'a montré Jean Starobinski dans La mélancolie au miroir : «L'expérience affective de la mélancolie, si souvent dominée par le sentiment de la pesanteur, est

\footnotetext{
23 PANERO, Leopoldo María, Buena Nueva del desastre (2002), in Poesía completa (2000-2010), op. cit., p. 118.

${ }^{24}$ Par exemple: Ob frente que boquea / sobre la página, dejando / como Pulgarcito un rastro de saliva. PANERO, Leopoldo María, Erección del labio sobre la página (2004), ibid, p. 193.

${ }^{25}$ PANERO, Leopoldo María, Danza de la muerte (2004), ibid, p. 221.

${ }^{26}$ Ibid., p. 215.
} 
inséparable de la représentation d'un espace hostile, qui bloque ou englue toute tentative de mouvement ${ }^{27}$. Dans l'œuvre panérienne, de façon étrange, le poème devient le réceptacle de tous les éléments qui tombent : y cae / la nieve sobre el hombre / y cae / la lluvia contra el hombre / y cae el hombre / a los pies de la página ${ }^{28}$. Ne pouvant rester en suspension, ni se charger de sens autres, les mots finissent par sombrer dans l'espace de la page, l'envol poétique leur étant refusé : page et espace poématique se confondent pour délimiter un lieu tombal qui scelle le destin d'une écriture mélancolique. Celle-ci ne parvient plus à créer un équilibre, à maintenir une tension qui serait l'assurance d'une parole qui puisse se prolonger, aussi tout se voit aspiré dans l'espace du poème. Si au XIX ${ }^{\mathrm{e}}$ siècle, les Romantiques, portés par un sentiment mélancolique, pouvaient se contempler dans un paysage escarpé, ou de ruines, le moi panérien n'a que la page ou le miroir sans tain pour se voir et se trouver, mais la rencontre avec soi ou avec une altérité poétique ne peut avoir lieu. Dans les recueils écrits entre 1975 et 1995, le moi panérien se définissait à travers une identité éclatée, une scission du poète avec l'être, mais que de multiples dédoublements et jeux de masques littéraires parvenaient à combler, en constituant une identité autre, celle d'un moi poétique ${ }^{29}$; en revanche, dans les derniers recueils, le moi semble dépossédé de lui-même, au bord de la disparition ou déjà mort.

\title{
Signe ultime du moi : le crapaud
}

Double inversé de la rose, comme le rappellent les binômes antithétiques beauté/laideur, ciel/terre, divin/infernal, solaire/lunaire et comme le notifie l'inversion des voyelles dans les deux substantifs rosa/sapo, le crapaud porte lui aussi des résonances littéraires que Leopoldo María Panero connaît, entre autres Tristan Corbière et son poème «Le crapaud» dans Les amours jaunes, celui de Victor Hugo dans La légende des siècles, ou encore celui d'Apollinaire dans Les saisons... L'on rappellera que L. M. Panero avait consacré un poème aux crapauds, dans une longue succession de quatorze strophes courtes, comme autant de sauts, qui traçaient leur histoire ${ }^{30}$. À présent, le crapaud fait corps avec le moi poétique; attribut des morts et des sorcières, le crapaud accompagne le moi panérien dans l'acte d'écrire. Signe maléfique, l'animal fait de la page ou du poème son espace, le transformant ainsi en un lieu sombre :

\author{
Oh tú mi soledad \\ amarillo ser de la página \\ húmedo silencio \\ que aquí florece \\ al otro lado de la vida \\ donde unos seres hablan y se mueven \\ al otro lado del espejo \\ sobre el poema \\ en donde crece la hierba
}

\footnotetext{
27 STAROBINSKI, Jean, La mélancolie au miroir. Trois lectures de Baudelaire, Paris, Julliard, 1989, p. 41.

${ }^{28}$ PANERO, Leopoldo María, Danza de la muerte (2004), in Poesía completa (2000-2010), op. cit., p. 212.

${ }^{29}$ Pour cet aspect, je me permets de renvoyer à ma thèse soutenue en 1999 : «L'œuvre poétique de Leopoldo María Panero : la quête d'une voix ", sous la direction de M-C. Zimmermann, Paris IV Sorbonne.

${ }^{30}$ PANERO, Leopoldo María, Últimos poemas, in Poesía (1970-1985), Madrid, Visor, 1986, p. 254.
} 


\author{
y mueren los sapos \\ lentamente, oh amarilla \\ cruz del odio y de la vida. ${ }^{31}$
}

Sorte de double du moi poétique, le crapaud opère comme la signature dissonante qui jalonne les recueils. Face à une poésie qui n'a plus lieu d'être dans un monde hostile, écrire revient à cracher et baver : El sapo sobre el poema / deja un rastro de baba / un animal azul I y un testamento de saliva ${ }^{32}$, poème placé sous la référence en exergue à Tristan Corbière «Bonsoir - ce crapaud-là c'est moi »-. De par leur rapprochement sonore, le verbe «escupir» se substitue à celui de "escribir», conférant ainsi à l'acte créateur une connotation de violence et de rejet, comme l'annonce le titre du recueil Escribir como escupir de $2008^{33}$. Mais pour le poète, les mots ne peuvent plus se former, toute construction langagière devient impossible: ne lui reste alors que le crachat, la salive, sécrétion qui rappelle la bave du crapaud, pour dire sa haine et sa désillusion. L. M. Panero porte jusqu'à sa limite la correspondance entre écrire et cracher, se désignant ainsi comme le dernier maillon d'une lignée d'écrivains qui en Espagne, ont dû se confronter à une âpre réalité historique : déjà dans le recueil Last River together, de 1980, le poète reprenait à son compte pour les amplifier en les plaçant sous un signe blasphématoire, les vers connus de Luis Cernuda, expression d'une violente répulsion envers l'Espagne: Escribir en España no es llorar, es beber, / es beber la rabia del que no se resigna / a morir en las esquinas, es beber y mal / decir, blasfemar contra España / contra este país sin dioses pero con / estatuas de dioses, es / beber en la iglesia con música de órgano ${ }^{34}$. Derrière ces vers où l'on entend la voix cernudienne - Escribir en España no es llorar, es morir ${ }^{35}$ - résonnent aussi d'autres paroles qui témoignent de la longue histoire des relations difficiles entre société et littérature, celles de Mariano José de Larra qui se suicida: Escribir como escribimos en Madrid es tomar una apuntación, es escribir en un libro de memorias, es realizar un monólogo desesperante y triste para uno solo. Escribir en Madrid es llorar, es buscar voz sin encontrarla, como en una pesadilla abrumadora y violenta. Porque no escribe uno siquiera para los suyos. ¿Quiénes son los suyos? ¿Quién oye aquỉ ${ }^{36}$ ? À travers ce dialogue par-delà le temps, une même préoccupation unit les poètes, une seule et même question : comment définir l'acte d'écrire quand celui-ci se voit mis à mal par la société et sa morale conservatrice. C'est pourquoi de l'un à l'autre, entend-on ainsi une autre voix, engagée mais tout aussi discordante, celle de Blas de Otero qui n'hésite pas à affirmer : Escribo como escupo. Contra el suelo / (ob esos poetas cursis, con sordina, / hijos de sus papás) y contra el cielo ${ }^{37}$. Malgré l'écart esthétique qui les sépare, car comme l'a souligné la critique, les Novísimos semblent se démarquer de la poésie précédente, poésie engagée puis sociale, entre L. M. Panero et Blas de Otero se noue un fil ténu de résistance contre la norme et la règle. Néanmoins, se sentant encore plus seul et en fin de lignée poétique, L. M. Panero ne voit plus le crachat comme acte subversif, il est à présent la manifestation d'une langue qui se liquéfie, la sécrétion dans laquelle le langage se dissout :

\footnotetext{
31 PANERO, Leopoldo María, Conjuros contra la vida (2008), in Poesía completa (2000-2010), op. cit., p. 486.

32 PANERO, Leopoldo María, Teoría del miedo (2000), ibid, p. 57.

33 PANERO, Leopoldo María, Escribir como escupir (2008), ibid, p. 445.

${ }^{34}$ PANERO, Leopoldo María, «La canción del croupier del Mississipi », in Poesía (1970-1985), Madrid, Visor, 1986, p. 188.

35 CERNUDA, Luis, "A Larra con unas violetas", Las nubes, in La realidad y el deseo (1924-1962), Madrid, Fondo de Cultura Económica, 1988, p. 145-146.

${ }^{36}$ LARRA, Mariano José de, "Horas de invierno », in El español, $\mathrm{n}^{\circ} 420,25$ de diciembre de 1836. Consultable en ligne sur le site cervantesvirtual.

${ }^{37}$ OTERO, Blas de, « Y el verso se hizo hombre », in Ancia, Madrid, Visor, 1958.
} 


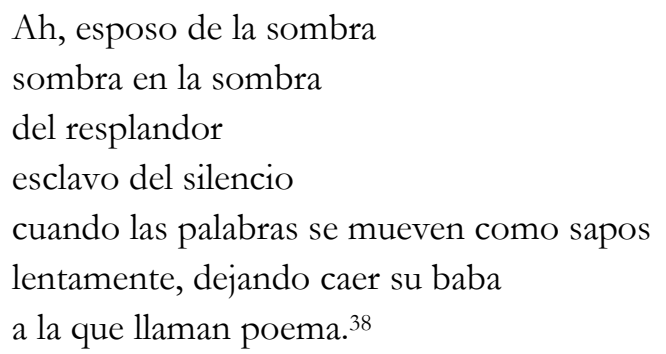

Le verbe créateur « escribir » a disparu, pour laisser place à celui de « escupir » qui suppose un déséquilibre entre pulsion de vie et pulsion de mort; écrire implique un processus de dissolution et de dégradation qui ébranle tout équilibre harmonieux : $O h$ mano cruel que escupe / sobre la tumba del poema / sobre la lápida de letras y de sílabas / indestructibles ${ }^{39}$. Certes, les lettres et les syllabes gravées dans la pierre se veulent éternelles, mais elles ne forment plus de mot, privées ainsi de tout sens. Salive, bave, urine, sperme, larme, autant de sécrétions qui se substituent aux mots et au langage, métonymies d'un poète devenu corps écrivant.

Il me semble qu'en traversant la mer pour rejoindre les Canaries, le poète a franchi sur le mode symbolique, le Styx poétique qui le conduit vers un territoire habité par la mort et l'oubli. Comme le montrent les poèmes des derniers recueils, cette traversée, vitale dans un premier temps, a supposé un deuil, un renoncement difficile à assumer qui a fait naitre le sentiment de mélancolie. Par la suite, la voix s'assombrit davantage, et se charge d'une noirceur poétique qui l'emmène vers un ailleurs où il sera difficile de demeurer. Nombreux sont les critiques qui évoquent une voix venant non pas d'un au-delà mais d'un temps et d'un lieu apocalyptiques, voix poétique à la limite de la voix humaine : « la poesía de Panero sería en conjunto ya no la última palabra, sino con mayor propiedad la palabra póstuma. Que esa palabra es póstuma, posterior al lenguaje, a la vida, al mundo, y desde luego dicha tras la muerte del sujeto es algo que está dicho reiteradamente en la escritura de Panero ${ }^{40} \%$.

Si écriture et biographie entretiennent des liens souvent difficiles à démêler, s'il est vrai par ailleurs que l'acte poétique suppose déjà une dépossession de soi, qu' «écrire implique », pour reprendre les propos d'André Green «une dissociation entre le sujet écrivant et le sujet d'écriture ${ }^{41}$, qu'en est-il du moi, ou du poète, de l'homme enfin dans la poésie de Leopoldo María Panero ? De par sa dimension mortifère, son écriture révèle une perception sensible de la mort qui génère, en même temps que confrontation et fascination, angoisse et terreur : les mots « espanto » «miedo» n’ont jamais été aussi présents. Si le sujet écrivant, soit le poète, ne semble plus habité par la vie, il résiste néanmoins aux assauts ou soubresauts d'une vie dépourvue de sens; en revanche et de façon contradictoire, c'est dans et par l'écriture qu'il ne cesse de se donner la mort. Si comme Panero le dit, écrire permet de repousser le suicide, paradoxalement mais aussi inexorablement, l'espace du poème devient le lieu de la mort à soi. C'est ainsi que le poème, la page apparaissent comme le dernier lieu où demeurer, faisant de vivre un synonyme de mourir ; en écrivant, le poète ne cesse de s'appréhender comme mort et s'enferme dans une écriture qui l'asphyxie. La poésie n'est plus salvatrice - en supposant qu'elle l'ait été-, pas plus que

\footnotetext{
38 PANERO, Leopoldo María, Esquizofrénicas o La balada de la lámpara azul (2004), in Poesía completa (20002010), op. cit., p. 250.

${ }^{39}$ PANERO, Leopoldo María, Erección del labio sobre la página (2004), ibid, p. 194.

40 BLESA, Túa, prólogo a la edición de Poesía completa (2000-2010), op. cit., p. 13.

41 GREEN, André, La Lettre et la Mort. Promenade d'un psychanalyste à travers la littérature: Proust, Shakespeare, Conrad, Borges..., Paris, Denoël, 2004, p. 98.
} 
cathartique : s'il ne reste à l'homme que l'écriture, celle le conduit au plus près de sa mort. Dans ce huis clos avec la poésie, les mots se répètent, les mêmes images reviennent mettant en évidence une sorte d'asséchement de l'imaginaire qui ressasse les mêmes obsessions, la tombe, sépulture, croix, cadavre, néant et autres signes qui connotent la mort, le ver, les os... sans jamais verser dans les vanités baroques. De recueil en recueil, le moi ne cesse d'affirmer sa mort, en même temps que celle de la poésie, sans jamais renoncer à écrire dans la mesure où il n'y a plus d'adéquation entre désir et réalité, entre vie et écriture, alors ne restent que les mots, ou ce qu'il en reste, pour dire le désastre : Oh tú poesía del desastre / y dulzura atroz del fin / siempre recomenzado / porque el llanto ya no existe ${ }^{42}$.

Pulsion de vie et pulsion de mort maintiennent une tension, certes encore créatrice, mais porteuse de la souffrance et du dénuement d'un être confronté à ses obsessions, confronté à une mort qui devient une échéance proche et palpable ${ }^{43}$ :

\author{
Moriré sin pensar sobre la página, \\ Mudo como un cadáver \\ En el secreto del bosque \\ Y sobre la página flotan los ruiseñores \\ Vencidos por la palabra, \\ Tambaleantes mejor que un borracho \\ Cayendo en pie sobre la página. ${ }^{44}$
}

42 PANERO, Leopoldo María, XII, Páginas de excremento o dolor sin dolor (2008), in Poesía completa (2000-2010), op. cit., p. 478.

${ }^{43}$ Le colloque a eu lieu les 28 et 29 novembre 2013, et Leopoldo María Panero est mort le 5 mars 2014.

${ }^{4}$ PANERO, Leopoldo María, XLV, Sombra (2008), in Poesía completa (2000-2010), op. cit., p. 375. 